\title{
Recurrent copy number alterations in young women with breast cancer
}

\author{
Chen Chi ${ }^{1,2}$, Leigh C. Murphy ${ }^{1,3}$ and Pingzhao $\mathrm{Hu}^{1,2,4}$ \\ ${ }^{1}$ Department of Biochemistry and Medical Genetics, University of Manitoba, Winnipeg, Manitoba, Canada \\ ${ }^{2}$ The George and Fay Yee Centre for Healthcare Innovation, University of Manitoba, Winnipeg, Manitoba, Canada \\ ${ }^{3}$ Research Institute of Oncology and Hematology, Cancer Care Manitoba, Winnipeg, Manitoba, Canada \\ ${ }^{4}$ Department of Electrical and Computer Engineering, University of Manitoba, Winnipeg, Manitoba, Canada \\ Correspondence to: Pingzhao Hu, email: pingzhao.hu@umanitoba.ca
}

Keywords: recurrent copy number alterations; breast cancer; young women; risk genes; graph algorithm

Received: September 07, $2017 \quad$ Accepted: January 24, $2018 \quad$ Published: January 29, 2018

Copyright: Chi et al. This is an open-access article distributed under the terms of the Creative Commons Attribution License 3.0 (CC BY 3.0), which permits unrestricted use, distribution, and reproduction in any medium, provided the original author and source are credited.

\section{ABSTRACT}

Breast cancer diagnosis in young women has emerged as an independent prognostic factor with higher recurrence risk and death than their older counterparts. We aim to find recurrent somatic copy number alteration (CNA) regions identified from breast cancer microarray data and associate the CNA status of the genes harbored in the regions to the survival of young women with breast cancer.

By using the interval graph-based algorithm we developed, and the CNA data consisting of a Discovery set with 130 young women and a Validation set with 125 young women, we identified 38 validated recurrent CNAs containing 39 protein encoding genes. CNA gain regions encompassing genes CAPN2, CDC73 and ASB13 are the top 3 with the highest occurring frequencies in both the Discovery and Validation dataset, while gene SGCZ ranked top for the recurrent CNA loss regions. The mutation status of 9 of the $\mathbf{3 9}$ genes shows significant associations with breast cancer specific survival. Interestingly, the expression level of 2 of the 9 genes, ASB13 and SGCZ, shows significant association with survival outcome. Patients with CNA mutations in both of these genes had a worse survival outcome when compared to patients without the gene mutations. The mutated CNA status in gene ASB13 was associated with a higher gene expression, which predicted patient survival outcome. Together, identification of the CNA events with prognostic significance in young women with breast cancer may be used in genomic-guided treatment.

\section{INTRODUCTION}

Although young women only account for $7 \%$ of all breast cancers, it is the most common cancer among young females [1]. Yet, young age at diagnosis of breast cancer has emerged as an independent factor for higher recurrence risk and death in various studies [2-6]. Breast cancer in young women has been described to have more biologically aggressive tumours (basal and HER2-enriched subtypes) than in older counterparts, which has been associated with a poorer prognosis [6]. Several factors influence poor prognosis in the young subgroup, such as higher tumour grade at diagnosis, high tumour proliferation, increased expression of HER-2 (ERB-B2) and reduced expression of both estrogen (ER) and progesterone receptor (PR) [7]. These women often struggle with life issues that are either absent or much less severe in older women, such as the possibility of early menopause and effects on fertility. While clinicopathologic differences point to underlying biological differences between breast tumours found in younger versus older women, limited studies have documented age-related changes at the molecular level.

Cancer progression is impelled by the accumulation of somatic genetic mutations, which consists of single nucleotide substitutions, translocations and somatic mutations [8]. Somatic mutations are non-heritable alterations to the 
human genome that occur spontaneously in somatic cells, which is often due to DNA replication error or chemical/ ultraviolet (UV) radiation. Copy number alterations (CNA) are somatic changes in the copy numbers of a DNA sequence that arise during the process of cancer development. They consist of changed chromosome structure in the form of gain or loss in copies of DNA segments, and are prevalent in many types of cancer [9]. Investigating these genomic alterations in breast cancer patients can not only offer valuable insights into breast cancer pathogenesis and discover potential biomarkers, but also provide novel drug targets for better therapeutic treatment options [10]. Several cytogenetic and array-based studies have detected recurrent alterations linked with certain cancer types, and have found CNAs to be a particularly common genetic mutation in cancer $[11,12]$. In addition, some of these CNAs have resulted in the discovery of disease causal genes and novel therapeutic targets, and have been strongly associated with clinical phenotypes [13-16]. For example, the use of vemurafenib to inhibit BRAF V600E mutation has shown remarkably improved survival in melanoma patients [17]. In another study, treatment with tyrosine kinase inhibitors for EGFR in lung cancer has also shown great success [18].

Since CNAs often encompass genes, it is suspected that they may greatly influence gene expression within the CNA regions. Indeed, several studies have reported a correlation between CNA and the average global expression levels of genes located within the copy number variable chromosomal regions. For instance, one group has shown that in tumour formation from an immortalized prostate epithelial cell line, $51 \%$ of genes with increased expression were mapped to DNA gain regions and $42 \%$ of genes with decreased expression were mapped to DNA loss regions [19]. This was further supported by another group working with breast tumour cell lines, noting that DNA copy number influences gene expression across a range of CNAs, with $62 \%$ of amplified genes resulting in moderately or highly elevated expression of the genes within the amplified regions [20].

Therefore, investigation of CNAs offers the potential to gain insight into the underlying genetic composition of breast tumours in young women. Mining genome-wide profiles will help find breast cancer genes and pathways with strong potential for prognostic significance as a function of age. Given that approximately $40-50 \%$ of young breast cancer patients relapse after 5 years [21], these agespecific signatures could also serve as a treatment decision tool to identify young patients that would gain more benefit from particular adjuvant therapies.

\section{RESULTS}

\section{Clinical characteristics}

The young patients with breast cancer in the Discovery and Validation Data sets retrieved from the
Molecular Taxonomy of Breast Cancer International Consortium (METABRIC) [22] have very similar distribution in age, menopausal status, tumour grade, tumour size, ER, PR expression and HER2 expressions $(p>0.05)$ (Table 1). On the other hand, the two sets have statistically significant differences in the tumour stage, with young patients in the Discovery set having a much higher prevalence in stage 0 compared to the Validation set $(43.1 \%$ vs $0.8 \%)$ and PAM50 subtypes $(p<0.05)$. However, an overall pattern of the basal subtype being the most frequent amongst young patients is apparent in both the Discovery and Validation dataset. It must be indicated that there are 50 patients in Validation set without stage information, which may affect the analysis of difference in distribution of stages between the two sets. Since our focus is only on those Discovery set CNA candidates that are validated in the Validation set, the stage difference is unlikely to be driving CNA selection. Furthermore, it is our intention to investigate whether tumours in young women share commonalities in genetic alterations, regardless of stages and subtypes.

\section{Identification of recurrent $\mathrm{CNA}$ regions}

Figure 1 shows the analysis flowchart to identify age-related recurrent CNA regions using our maximal clique-based recurrent CNA detection algorithm. In the METABRIC Discovery cohort 867 of the total 997 patients are classified into the old age group ( $\geq 45$ years old) and 130 patients into the young age group. In the Validation cohort 870 of the total 995 patients are classified into the old age group and 125 patients into the young age group. After applying filtering criteria (retaining CNA data that was generated by $\geq 10$ probes and having a CNA size of at least $1 \mathrm{~kb}$ ), for the old age cohort in the Discovery set, there are 96,503 and 47,943 individual patient level CNA gain and loss regions respectively. For the young age cohort, there are 14,957 and 6,373 individual patient level CNA gain and loss regions, respectively.

Upon filtering for recurrent CNA regions of at least 1 kilobase $(\mathrm{kb})$ in size and having at least 5 patients per recurrent region identified from the recurrent CNA calling algorithm, there are a total of 1,086 recurrent CNA gain regions (554 of the 1,086 gain regions encompassing protein encoding genes) and 439 recurrent CNA loss regions (202 of the 439 loss regions encompassing protein encoding genes). These regions are uniquely found in the young age group and form the young-specific recurrent gain and loss regions in the Discovery set.

Validation testing is then performed using the Validation set, which contains 995 patients. All filtering criteria and algorithm implementations follow the same procedure as the Discovery dataset analysis. For recurrent CNA gain regions, a total of 81 of the 1,086 regions have been validated (found in both the Discovery and Validation datasets), in which 30 regions have encompassed 29 
Table 1: Clinical characteristics table comparing the METABRIC discovery dataset and validation dataset for young patients only

\begin{tabular}{|c|c|c|c|}
\hline Characteristic & Discovery Young & Validation Young & ${ }^{\dagger} P$-value \\
\hline Age* & $40(36,43)$ & $40(37,43)$ & 1 \\
\hline Menopausal Status & & & 0.5 \\
\hline Pre & $127\left(97.7 \%{ }^{* *}\right)$ & $125(100 \%)$ & \\
\hline Post & $2(1.5 \%)$ & $0(0.0 \%)$ & \\
\hline Subtype & & & $<0.001$ \\
\hline Normal & $11(8.5 \%)$ & $25(20 \%)$ & \\
\hline LumA & $41(31.5 \%)$ & $18(14.4 \%)$ & \\
\hline $\operatorname{LumB}$ & $20(15.4 \%)$ & $9(7.2 \%)$ & \\
\hline Her2 & $16(12.3 \%)$ & $21(16.8 \%)$ & \\
\hline Basal & $42(32.3 \%)$ & $52(41.6 \%)$ & \\
\hline Grade & & & 0.98 \\
\hline 1 & $7(5.4 \%)$ & $6(4.8 \%)$ & \\
\hline 2 & $37(28.5 \%)$ & $34(27.2 \%)$ & \\
\hline 3 & $86(66.1 \%)$ & $81(64.8 \%)$ & \\
\hline Stage & & & $<0.001$ \\
\hline 0 & $56(43.1 \%)$ & $1(0.8 \%)$ & \\
\hline 1 & $25(19.2 \%)$ & $28(22.4 \%)$ & \\
\hline 2 & $42(32.3 \%)$ & $35(28.0 \%)$ & \\
\hline 3 & $7(5.4 \%)$ & $11(8.8 \%)$ & \\
\hline 4 & $0(0.0 \%)$ & $0(0.0 \%)$ & \\
\hline ER-expr & & & 0.09 \\
\hline+ & $74(56.9 \%)$ & $57(45.6 \%)$ & \\
\hline- & $56(43.1 \%)$ & $68(54.4 \%)$ & \\
\hline PR-expr & & & 0.78 \\
\hline+ & $55(42.3 \%)$ & $56(44.8 \%)$ & \\
\hline- & $75(57.7 \%)$ & $69(55.2 \%)$ & \\
\hline Her2-expr & & & 0.27 \\
\hline+ & $22(16.9 \%)$ & $29(23.2 \%)$ & \\
\hline- & $108(83.1 \%)$ & $96(76.8 \%)$ & \\
\hline Tumour Size* (mm) & $22(16,30)$ & $(17,30)$ & 1 \\
\hline
\end{tabular}

"For continuous variables (Age, Tumour size), quantiles (50th percentile (25th percentile, 75th percentile)) were presented. ${ }^{\dagger} P$-values were determined by Wilcoxon rank sum test for continuous variables and Fisher's exact test for categorical variables.

${ }^{* *}$ The proportion was obtained by dividing the total number of patients.

unique protein encoding genes (Table 2). For recurrent CNA loss regions, a total of 25 of the 439 regions have been validated, in which 8 regions encompassed 10 unique protein encoding genes (Table 3 ). In total, 38 validated recurrent CNA regions with 39 protein encoding genes were identified, along with 51 validated recurrent gain CNA regions (Supplementary Table 1) and 17 validated recurrent loss CNA regions (Supplementary Table 2) that did not encompass any protein encoding genes.
Figure 2 shows an overview of how similar the cluster sizes (i.e. number of patients) are in the Discovery set versus the Validation set for all the identified young-specific recurrent CNA regions. It can be seen that for both gain and loss regions, cluster sizes in the Discovery and Validation datasets have a fairly linear relationship. For example, if $30 \%$ of the young patients in the Discovery set harbour a CNA region, it is likely that around $30 \%$ of patients in the Validation set will harbour that region as well. 

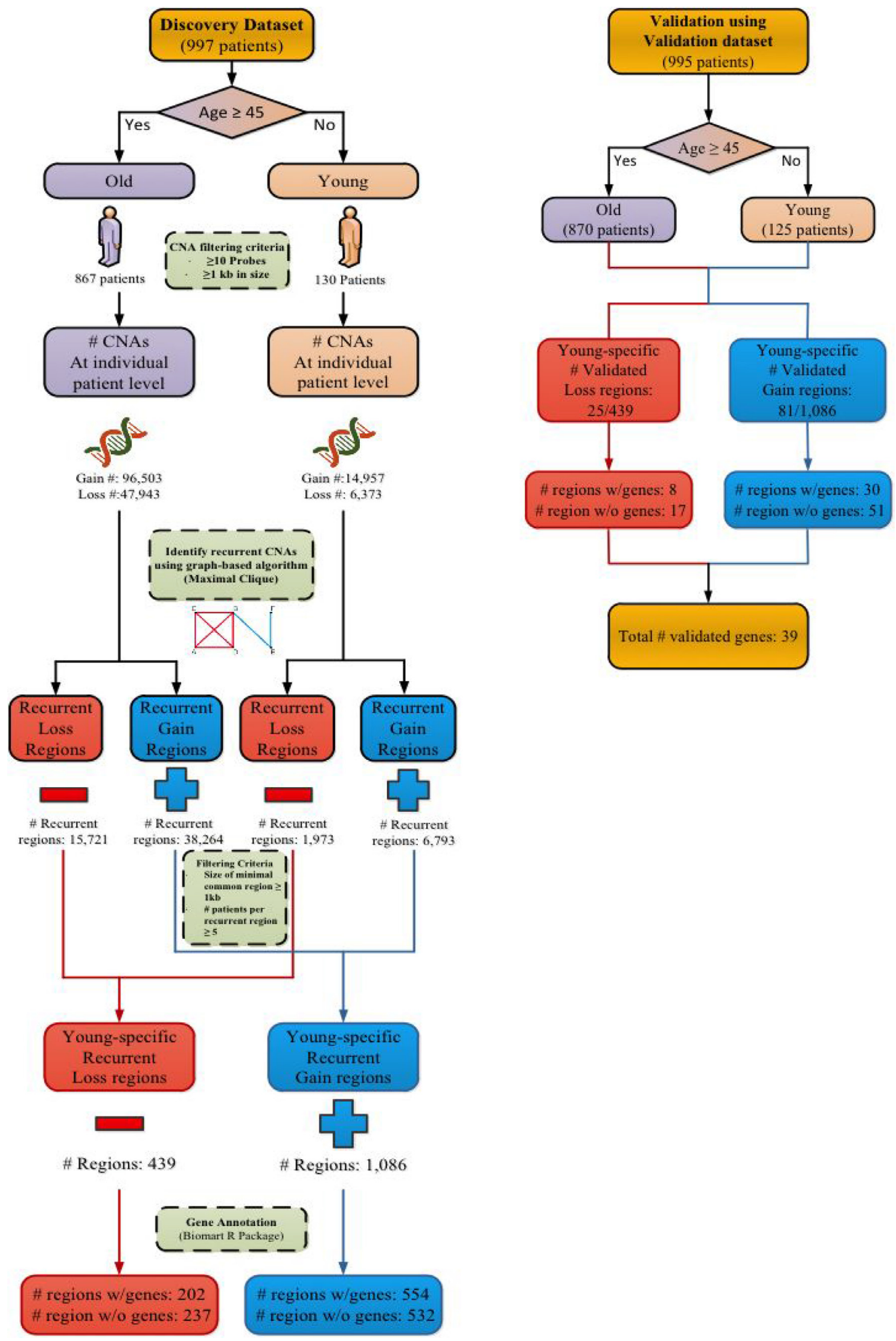

Figure 1: Analysis flowchart for identifying recurrent CNA regions. Recurrent CNA regions are identified from young and old patient cohorts in the Discovery set of METABRIC. The identified recurrent CNA regions are then validated in the Validation set of METABRIC. 
Table 2: Validated recurrent gain CNA regions with genes

\begin{tabular}{|c|c|c|c|c|c|c|c|c|c|}
\hline Chr & Inner Start & Inner End & Inner Size & Outer Start & Outer End & $\begin{array}{c}\text { Outer } \\
\text { Size }\end{array}$ & Gene Symbol & Size1 & Size2 \\
\hline 1 & 84551640 & 84565561 & 13921 & 84481190 & 84729446 & 248256 & SAMD13 & 5 & 6 \\
\hline 1 & 143607802 & 143609034 & 1232 & 143607067 & 143609055 & 1988 & PDE4DIP & 20 & 30 \\
\hline 1 & 191374290 & 191385577 & 11287 & 191359529 & 191405183 & 45654 & CDC73 & 40 & 50 \\
\hline 1 & 191385797 & 191402004 & 16207 & 191359529 & 191405183 & 45654 & CDC73 & 40 & 50 \\
\hline 1 & 222004315 & 222004925 & 610 & 221990859 & 222005526 & 14667 & CAPN2 & 48 & 47 \\
\hline 3 & 176423680 & 176427601 & 3921 & 176415397 & 176428705 & 13308 & NAALADL2 & 18 & 22 \\
\hline 3 & 176428607 & 176428705 & 98 & 176415397 & 176470832 & 55435 & NAALADL2 & 18 & 22 \\
\hline 5 & 22246497 & 22346803 & 100306 & 22194503 & 22414425 & 219922 & CDH12 & 12 & 11 \\
\hline 6 & 34625387 & 34634997 & 9610 & 34624907 & 34656516 & 31609 & SPDEF & 9 & 9 \\
\hline 7 & 134782461 & 134787038 & 4577 & 134782461 & 134792291 & 9830 & CNOT4 & 11 & 13 \\
\hline 7 & 142150844 & 142154230 & 3386 & 142150819 & 142154515 & 3696 & PRSS1 & 7 & 10 \\
\hline 8 & 40695071 & 40697114 & 2043 & 40693570 & 40699795 & 6225 & ZMAT4 & 17 & 16 \\
\hline 9 & 93166194 & 93261927 & 95733 & 93157333 & 93373420 & 216087 & NFIL3 & 5 & 7 \\
\hline 10 & 5737990 & 5742226 & 4236 & 5736767 & 5744158 & 7391 & ASB13 & 24 & 32 \\
\hline 10 & 14598341 & 14600566 & 2225 & 14477106 & 14629604 & 152498 & FAM107B & 19 & 27 \\
\hline 11 & 4931741 & 4932834 & 1093 & 4931741 & 4932966 & 1225 & MMP26; OR51A2 & 7 & 11 \\
\hline 12 & 180797 & 191614 & 10817 & 180797 & 195197 & 14400 & SLC6A12 & 14 & 24 \\
\hline 12 & 7895693 & 7897774 & 2081 & 7895693 & 7897774 & 2081 & SLC2A14 & 13 & 23 \\
\hline 12 & 7899067 & 7905082 & 6015 & 7899067 & 7909593 & 10526 & SLC2A14 & 13 & 23 \\
\hline 13 & 112354883 & 112363586 & 8703 & 112333434 & 112363586 & 30152 & C13orf35 & 10 & 7 \\
\hline 13 & 113356126 & 113365589 & 9463 & 113345036 & 113371998 & 26962 & ATP4B & 10 & 9 \\
\hline 17 & 43751830 & 43753351 & 1521 & 43722185 & 43756717 & 34532 & SKAP1 & 9 & 14 \\
\hline 17 & 45136676 & 45139395 & 2719 & 45134175 & 45142242 & 8067 & SLC35B1 & 21 & 18 \\
\hline 18 & 9549925 & 9575313 & 25388 & 9417006 & 9594232 & 177226 & PPP4R1 & 5 & 7 \\
\hline 18 & 43704001 & 43707399 & 3398 & 43703934 & 43707399 & 3465 & SMAD2 & 6 & 6 \\
\hline 19 & 40629439 & 40647918 & 18479 & 40620640 & 40702499 & 81859 & FFAR2 & 13 & 13 \\
\hline 19 & 60890917 & 60901410 & 10493 & 60890917 & 60904859 & 13942 & EPN1 & 8 & 11 \\
\hline 20 & 14741416 & 14743670 & 2254 & 14741416 & 14743754 & 2338 & MACROD2 & 8 & 10 \\
\hline 20 & 41215727 & 41219453 & 3726 & 41202818 & 41220578 & 17760 & PTPRT & 12 & 18 \\
\hline 22 & 20146692 & 20170596 & 23904 & 20145867 & 20170766 & 24899 & $\begin{array}{l}\text { PI4KAP2; } \\
\text { TMEM191C }\end{array}$ & 7 & 15 \\
\hline
\end{tabular}

The first seven columns represent the chromosome number, inner and outer start and end coordinates of the recurrent CNA region, and the size of the region in base pairs (hg18). The last three columns are the genes encompassed in each CNA region, followed by the sample size (no. of cases) in both Discovery and Validation dataset (Chr: Chromosome; Size 1: Discovery Cluster Size; Size 2: Validation Cluster Size).

\section{Annotation of the identified recurrent CNA regions}

We performed region-based variation annotation on the identified young-specific recurrent CNA regions (see Tables 2 and 3 and Supplementary Tables 1 and 2) with refGene using the software ANNOVAR (Annotate
Variation). The complete annotation information of the recurrent CNA regions is shown in Supplementary Table 3. Figure 3 shows the genome location distribution of our recurrent CNAs with respect to the encompassed regions. The majority of the CNAs are in non-coding regions (76\%) and $24 \%$ in coding regions. 
Table 3: Validated recurrent loss CNA regions with genes

\begin{tabular}{cccccccccc}
\hline Chr & Inner Start & Inner End & $\begin{array}{c}\text { Inner } \\
\text { Size }\end{array}$ & Outer Start & Outer End & $\begin{array}{c}\text { Outer } \\
\text { Size }\end{array}$ & Gene Symbol & Size1 & Size2 \\
\hline 2 & 97507180 & 97517476 & 10296 & 97507180 & 97520698 & 13518 & ANKRD36B & 5 & 5 \\
3 & 62243538 & 62257523 & 13985 & 62242606 & 62277516 & 34910 & PTPRG & 6 & 6 \\
4 & 59521 & 61566 & 2045 & 59521 & 64435 & 4914 & ZNF718; ZNF595 & 13 & 6 \\
7 & 38296343 & 38297866 & 1523 & 38295506 & 38297939 & 2433 & TRGV11 & 18 & 22 \\
8 & 14388851 & 14391732 & 2881 & 14385622 & 14391732 & 6110 & SGCZ & 23 & 18 \\
9 & 5027454 & 5029342 & 1888 & 5027454 & 5030334 & 2880 & JAK2 & 7 & 11 \\
10 & 89710114 & 89713882 & 3768 & 89708179 & 89713882 & 5703 & PTENP1; PTEN & 10 & 10 \\
17 & 21245986 & 21253816 & 7830 & 21227031 & 21271210 & 44179 & KCNJ12 & 15 & 9 \\
\hline
\end{tabular}

The first seven columns represent the chromosome number, inner and outer start and end coordinates of the recurrent CNA region, and the size of the region in base pairs (hg18). The last three columns are the genes encompassed in each CNA region, followed by the sample size in both Discovery and Validation dataset (Chr: Chromosome; Size 1: Discovery Cluster Size; Size 2: Validation Cluster Size).

In order to better visualize the mutation distribution of the 39 genes encompassed in the recurrent CNAs identified in the coding regions in both the Discovery and Validation young women group, an R package called the ComplexHeatmap was applied (Figure 4). From the heatmap, it can be observed that CNA gain regions encompassing genes $C A P N 2, C D C 73$ and $A S B 13$ are the top 3 most frequent in both Discovery and Validation dataset (young women age group), while gene $S G C Z$ ranks top for recurrent CNA loss regions in the two datasets.

\section{Expression quantitative trait locus analysis}

An overview of the expression levels for each of the identified young-specific genes across all the young patients samples in the Discovery (Figure 5A) and Validation datasets (Figure 5B) is provided as gene expression heatmaps. Further interrogation using logistic regression was performed to evaluate the statistical association between gene expression and CNA mutation status (Table 4). In total, 16 gain regions and 1 loss region show significant associations with their gene expression changes. However, the directionality of the association is ambiguous. Fourteen out of the 16 gain regions correlated with high gene expression while the other 2 gain regions (encompassing $M M P 26$ and $S P D E F$ ) were associated with low gene expression. For example, mutated gain CNA status in $A S B 13$ seems to lead to higher gene expression. On the other hand, the loss region encompassing PTEN was found to be associated with having high gene expression level.

\section{Survival analysis}

We further evaluate whether the expression levels of these genes are associated with disease-specific survival
(DSS) (Table 5). The expression levels of eight out of the 39 young-specific genes are significantly associated with survival outcome. A higher gene expression of genes CAPN2, NFIL3 and SLC35B1 was associated with a moderately worse survival outcome.

Of particular interest, the mutation status of two genes, $A S B 13$ (Figure 6A) and SGCZ (Figure 7D), was also significant in the Kaplan Meier survival analysis, which allows estimation of a survival curve over time. Patients with a mutated status in both of these genes resulted in a worse survival outcome when compared to patients without the gene mutations. Other genes found to be significant in the survival analysis include $A T P 4 B$ (Figure 6B), FFAR2 (Figure 6C) and PTPRT (Figure 6D), all encompassed within CNA gain regions. PTENP1 (Figure 7A), PTEN (Figure 7B), ZNF718 (Figure 7C) and ZNF595 (Figure 7E), all encompassed in CNA loss regions.

\section{Cancer-relevant candidate genes}

\section{PTEN (Phosphatase and tensin homolog)}

Results from our study show that the median survival time (i.e. half of the patients are expected to be alive) for young patients with a copy number loss in the PTEN gene region is $\sim 4$ years as opposed to $\sim 15$ years for those without. PTEN (cytoband 10q23.31) has been identified as a tumour suppressor which inhibits the PI3K/Akt/mTOR signalling pathways [23]. It has been shown to be one of the most frequently mutated genes in all cancer types, including that of breast, ovary, prostate, glioblastoma and lymphoma. Previous studies have observed that $40 \%$ of invasive breast cancers have a loss of PTEN heterozygosity, and that the loss of one gene copy is sufficient to disrupt cell signalling and cell growth control. It has also been 
suggested that carriers of the PTEN mutation are at higher risk of developing breast cancers at a younger age [24].

\section{SGCZ (sarcoglycan zeta)}

Our study shows that $\sim 16 \%$ of all young patients present a CNA loss mutation encompassing $S G C Z$, with a significantly shorter median survival time for young patients with this mutation of $\sim 6$ years in contrast to $\sim 15$ years for those without. $S G C Z$ (8p22) encodes a protein that is part of the sarcoglycan complex, which plays a role in connecting the inner cytoskeleton to the extracellular matrix, possibly maintaining membrane stability [25]. Although the exact function of $S G C Z$ in cancer is not well understood, loss of the chr8p region has been associated with several factors involved in cancer development and progression, such as the tumour having an aggressive histology, increased cell proliferation, and large size as
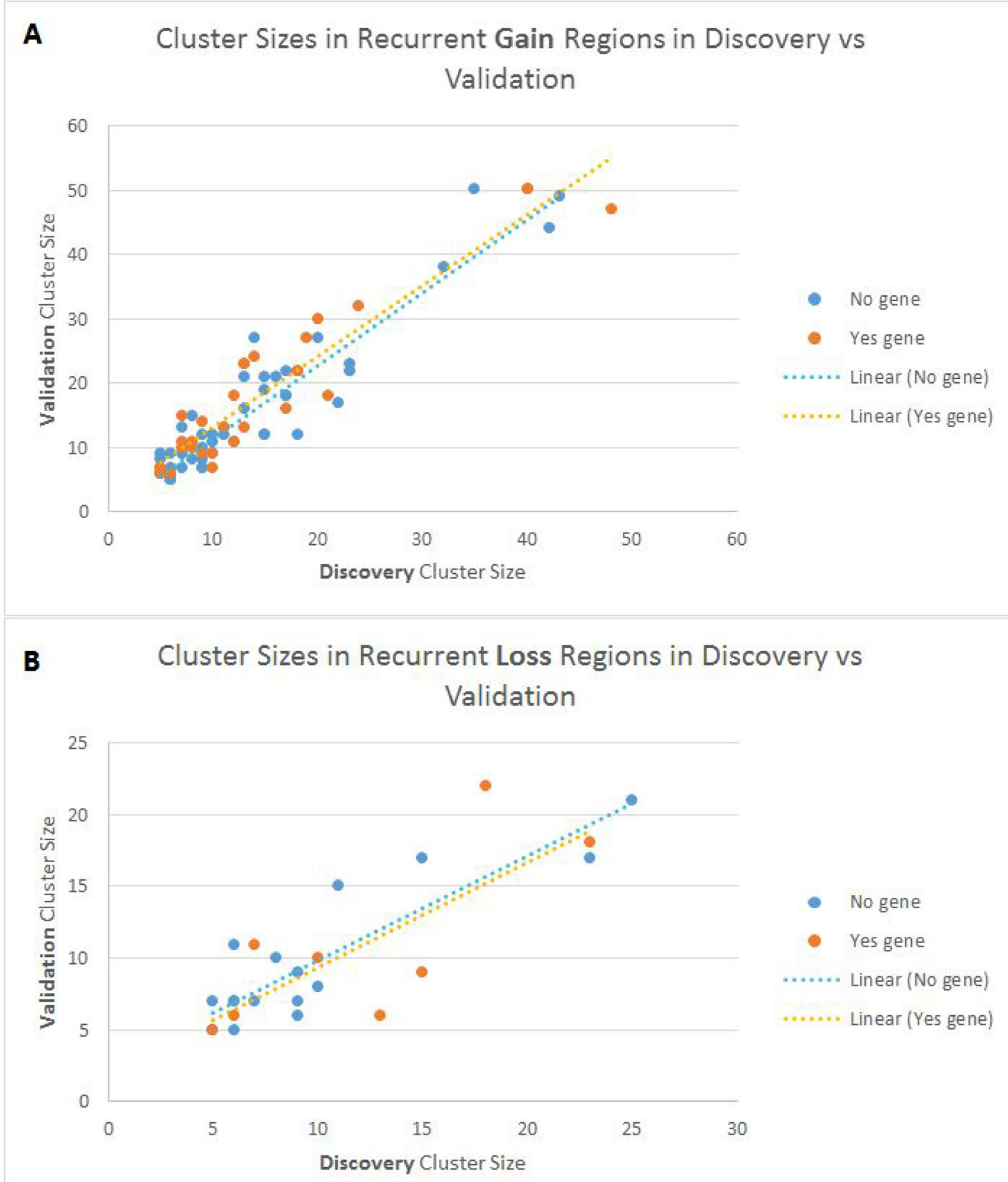

Figure 2: Scatter plot showing the cluster sizes of recurrent CNA regions in the discovery and validation sets. (A) Gain recurrent young-specific regions and (B) Loss recurrent young-specific regions. Each point on the plot represents a young-specific recurrent CNA region. Blue represents regions without genes, and orange represents regions with genes. 
well as the patients having increased early recurrence rate and mortality, and overall poor survival in young women. This region also contains the gene $D L C 1$ (deleted in liver cancer 1), which has been suggested to act as a tumour suppressor [26]. DLC1 encodes a GAP protein that inhibits the activation of Rho-GTPases, which are often associated with a loss of cell adhesion. DLC1 expression has been reported to be frequently lost in tumour cells, leading to a constitutive activation of the Rho-GTPases.

\section{CAPN2 (calpain 2)}

CAPN2 (cytoband 1q41) was the most frequent CNA gain mutation in our study, with $\sim 37-38 \%$ of all young patients harbouring a CAPN2 gain mutation. Calpains are calcium-activated intracellular proteases that have the ability to cleave cytoskeletal proteins, possibly playing a role in regulating cell invasion and migration [27]. A knockdown study of CAPN2 in breast tumour cells resulted in reduced cell migration, proliferation, as well as reduced Akt activation, increased FoxO nuclear localization and p27 expression [27]. It was suggested that CAPN2 promotes cell proliferation through the AktFoxO-p27 signalling pathway.

\section{NAALADL2 (N-acetyl-L-aspartyl-L-glutamate peptidase-like 2)}

Our study shows that $\sim 16 \%$ of all young patients present a CNA gain mutation encompassing NAALADL2. NAALADL2 is a member of the NAALADase protein family which act as matrix metalloproteases and have the ability to alter the tumour environment. Microarray studies have shown that NAALADL2 is often overexpressed in prostate and colon cancers and stimulates a migratory and metastatic phenotype. A proposed mechanism is that since NAALADL2 has been found to be basallocalized, it may enhance interaction of tumour cells with the extracellular matrix surrounding the tumour and provide a mechanism for the tumour cells to escape [28]. Subsequent survival analysis shows that patients with NAALADL2 overexpression have a $45 \%$ chance of surviving up to 5 years as opposed to $93 \%$ for patients with low NAALADL2 expression. It remained prognostic for recurrence rate even after correction for clinical variables such as tumour stage and grade. Expression array analyses also associated its overexpression to changes in the epithelial-to-mesenchymal transition (EMT) and cell adhesion pathways.

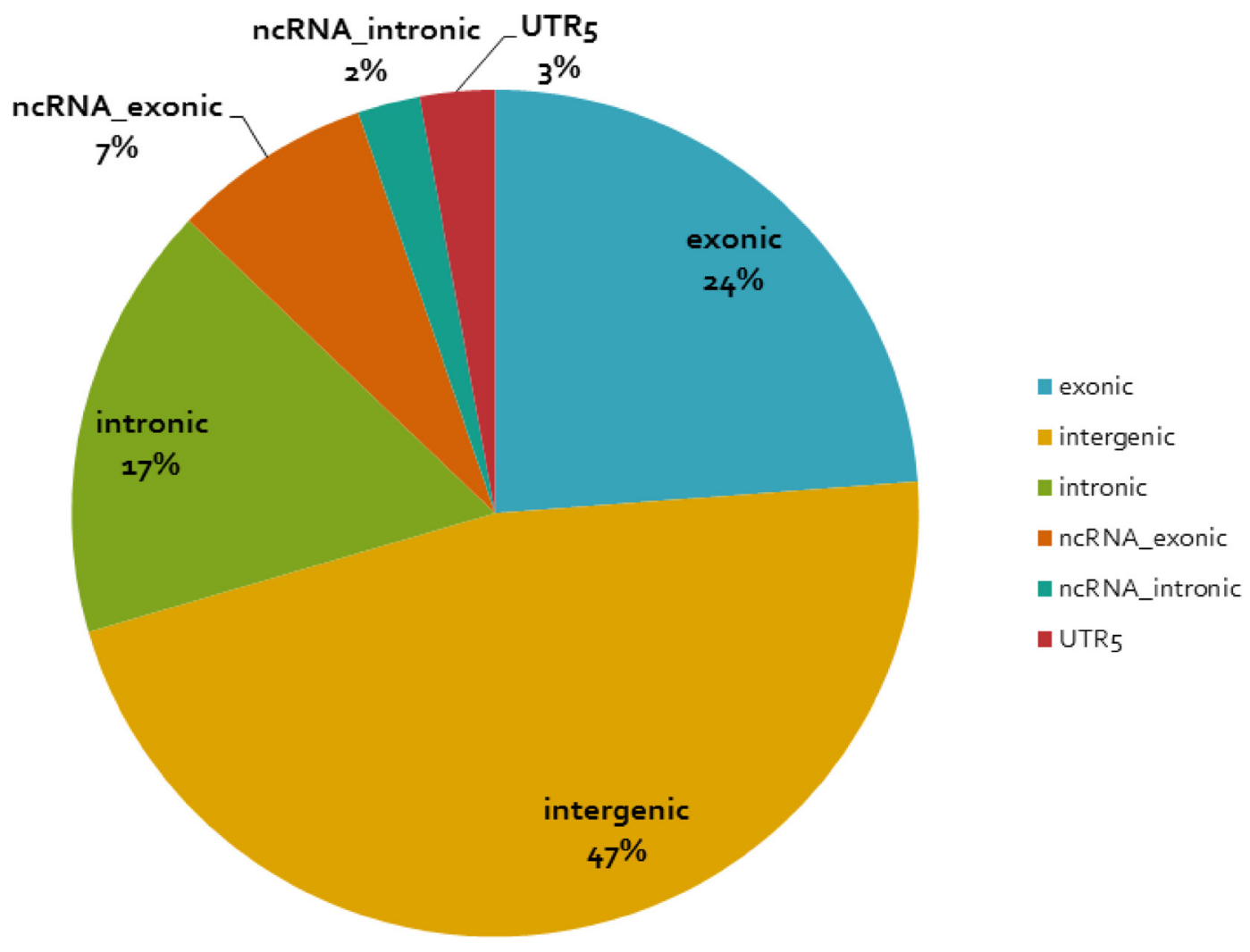

Figure 3: Distribution of the identified young-specific recurrent CNA regions with respect to the genome location. The functional annotation of the young-specific recurrent CNA regions is based on software ANNOVAR. 


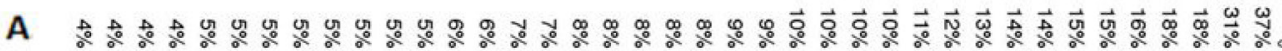

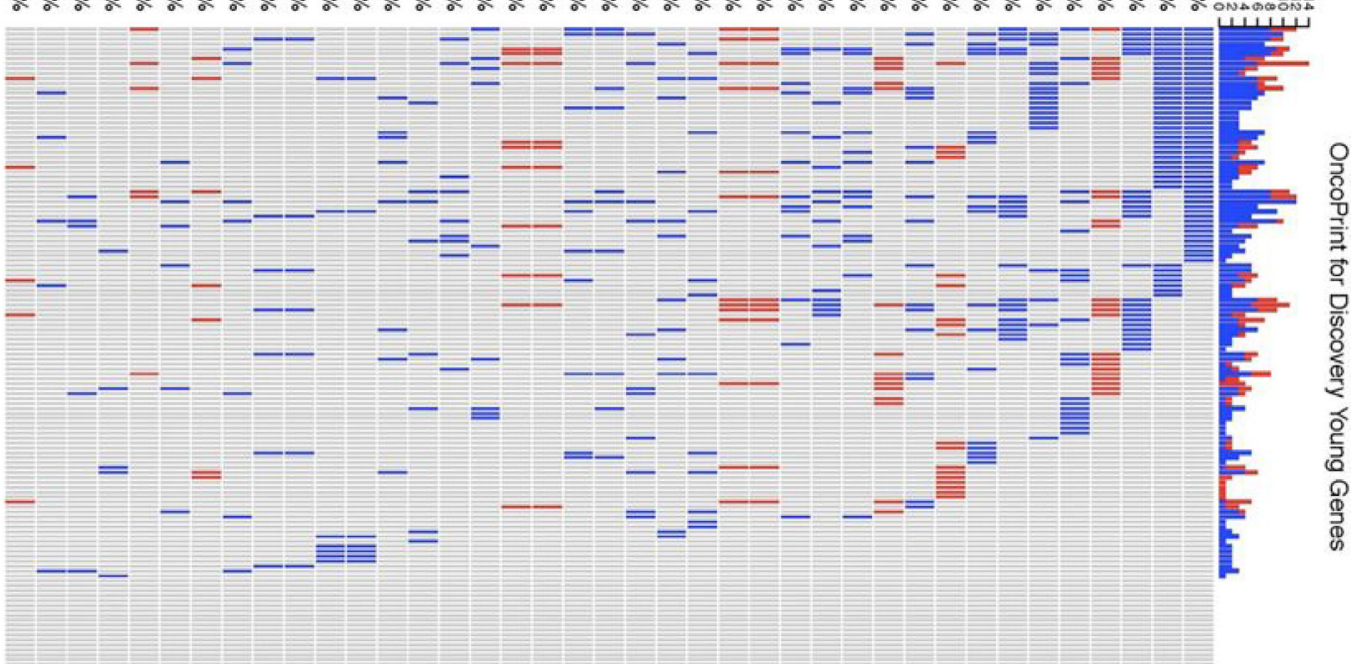

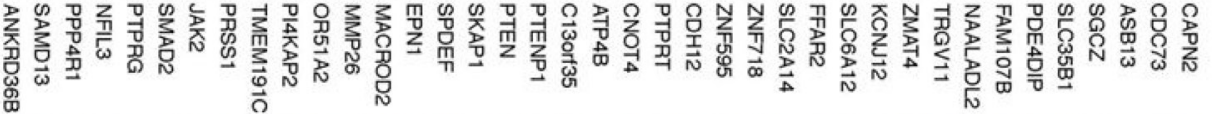

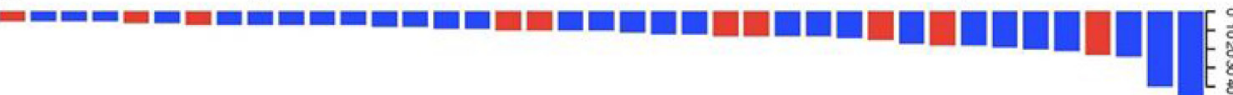

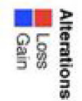

B

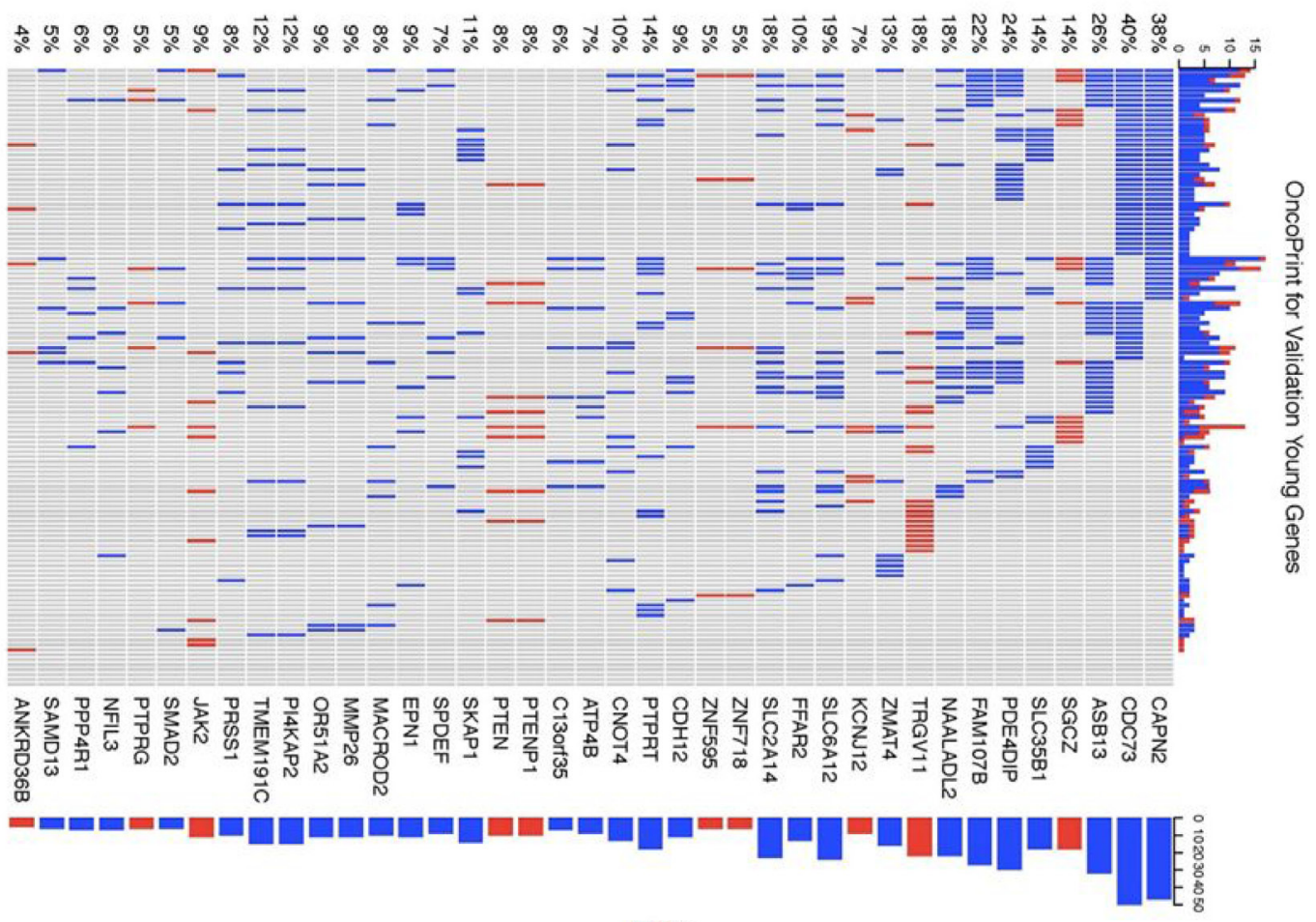

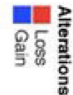

Figure 4: Heatmap of mutation distribution for genes identified in the recurrent young-specific CNA gain and loss regions. (A) Results from the Discovery dataset and (B) Results from the Validation dataset. Rows are sorted based on the frequency of the alterations in all young-specific samples and columns are sorted to visualize the mutual exclusivity across genes. Barplots at both sides of the heatmap show numbers of different alterations for each sample and for each gene. Red represents CNA loss mutations and blue represents CNA gain mutations. 

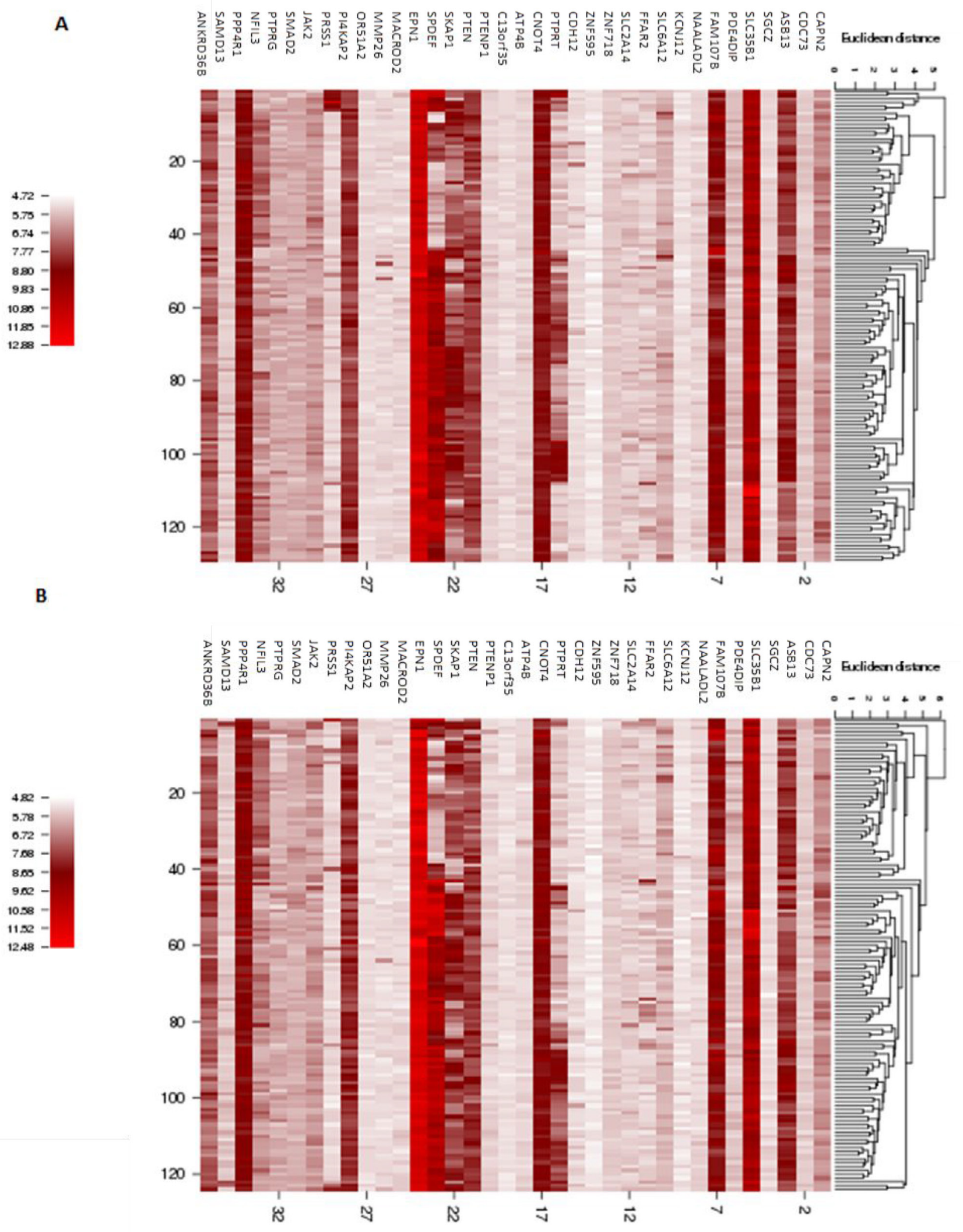

Figure 5: Heatmap of gene expression for the genes identified in the recurrent young-specific CNA regions in young breast cancer patients. (A) Results from the Discovery dataset (B) Results from the Validation dataset. Rows represent the gene expression levels for the genes identified in the recurrent young-specific CNA gain and loss regions (same order as in Figure 4 for comparison). Columns represent the young-specific samples in Discovery and Validation datasets. The higher the intensity of the red colour, the higher the gene expression level. 
Table 4: Logistic regression analysis between CNA mutation status and gene expression in combined dataset

\begin{tabular}{|c|c|c|c|c|c|}
\hline Gene Symbol & $\begin{array}{c}\text { Copy Number } \\
\text { State }\end{array}$ & $P$-value & Odds Ratio $^{\dagger}$ & $\begin{array}{c}\text { Discovery } \\
\text { Sample Size }\end{array}$ & $\begin{array}{l}\text { Validation } \\
\text { Sample Size }\end{array}$ \\
\hline ASB13 & Gain & 0.049 & $1.83(1.00-3.34)$ & 24 & 32 \\
\hline АТР4B & Gain & 0.021 & $3.51(1.21-10.17)$ & 10 & 9 \\
\hline CAPN2 & Gain & 0.00002 & $3.21(1.89-5.45)$ & 48 & 47 \\
\hline CDH12 & Gain & 0.026 & $2.87(1.14-7.24)$ & 12 & 11 \\
\hline CNOT4 & Gain & 0.0004 & 7.45 (2.46-22.49) & 11 & 13 \\
\hline EPN1 & Gain & 0.004 & $9.57(2.15-42.56)$ & 8 & 11 \\
\hline PDE4DIP & Gain & 0.002 & $2.73(1.45-5.15)$ & 20 & 30 \\
\hline PI4KAP2 & Gain & 0.031 & $2.81(1.10-7.14)$ & 7 & 15 \\
\hline PPP4R1 & Gain & 0.016 & $6.7(1.44-31.23)$ & 5 & 7 \\
\hline SLC35B1 & Gain & 0.001 & $19.19(6.54-56.27)$ & 21 & 18 \\
\hline SMAD2 & Gain & 0.022 & $6.06(1.30-28.22)$ & 6 & 6 \\
\hline SPDEF & Gain & 0.057 & $0.39(0.15-1.03)$ & 9 & 9 \\
\hline FAM107B & Gain & 0.083 & $1.79(0.93-3.46)$ & 19 & 27 \\
\hline MACROD2 & Gain & 0.085 & $2.73(0.87-8.55)$ & 8 & 10 \\
\hline MMP26 & Gain & 0.098 & $0.43(0.15-1.17)$ & 7 & 11 \\
\hline NFIL3 & Gain & 0.068 & $3.46(0.91-13.08)$ & 5 & 7 \\
\hline PTEN & Loss & 0.002 & $29.12(3.83-221.25)$ & 10 & 10 \\
\hline
\end{tabular}

Odds ratio is followed by its corresponding 95\% confidence interval in brackets.

Table 5: Cox proportional hazard analysis of disease (breast cancer) specific survival of gene expression in combined dataset (discovery and validation)

\begin{tabular}{lcccccccc}
\hline Genes & $\begin{array}{c}\text { CNA } \\
\text { types }\end{array}$ & $\boldsymbol{P}$-value & Hazard ratio $^{\dagger}$ & $\begin{array}{c}\text { Discovery } \\
\text { Sample } \\
\text { Size }\end{array}$ & $\begin{array}{c}\text { Validation } \\
\text { Sample } \\
\text { Size }\end{array}$ & Chr & InnerStart & InnerEnd \\
\hline ASB13 & Gain & 0.0001 & $0.54(0.39-0.73)$ & 24 & 32 & 10 & 5737990 & 5742226 \\
CAPN2 & Gain & 0.091 & $1.54(0.93-2.54)$ & 48 & 47 & 1 & 222004315 & 222004925 \\
NFIL3 & Gain & 0.009 & $1.58(1.13-2.23)$ & 5 & 7 & 9 & 93166194 & 93261927 \\
PDE4DIP & Gain & 0.027 & $0.37(0.16-0.89)$ & 20 & 30 & 1 & 143607802 & 143609034 \\
PTPRT & Gain & 0.0002 & $0.68(0.55-0.83)$ & 12 & 18 & 20 & 41215727 & 41219453 \\
SKAP1 & Gain & 0.0002 & $0.66(0.53-0.82)$ & 9 & 14 & 17 & 43751830 & 43753351 \\
SLC35B1 & Gain & 0.00005 & $1.88(1.39-2.55)$ & 21 & 18 & 17 & 45136676 & 45139395 \\
JAK2 & Loss & 0.007 & $0.43(0.24-0.79)$ & 7 & 11 & 9 & 5027454 & 5029342 \\
\hline
\end{tabular}

Hazard ratio is followed by its corresponding $95 \%$ confidence interval in brackets.

\section{Pathway enrichment analysis}

A pathway enrichment analysis using the ANNOVAR gene list (174 genes) via the Enrichr REACTOME database reveals a significant overrepresentation of phospholipid signaling (MTMR14,PTEN,PIP4K2A) and adherens junction $(\mathrm{CDH12}, \mathrm{CDH18}, \mathrm{CDH7})$ pathways $(p<0.05)$ in the identified young-specific recurrent CNA regions with genes. Both enriched pathways are highly relevant to cancer development and progression.

\section{Phospholipid signaling}

Aside from playing an important role in structural components, lipids also have a role in signalling processes $[29,30]$. These lipid molecules aggregate to form lipid 
rafts as highly specific platforms for cell signalling, carrying signals from activated growth factor receptors to the intracellular machinery [31]. These receptors recruit signalling effectors that induce cell proliferation and reduce cell death, dysregulation of which contributes to cancer development and progression. The phosphatidylinositol $(3,4,5)$-trisphosphate molecule, also known as PIP3, is

A

ASB13

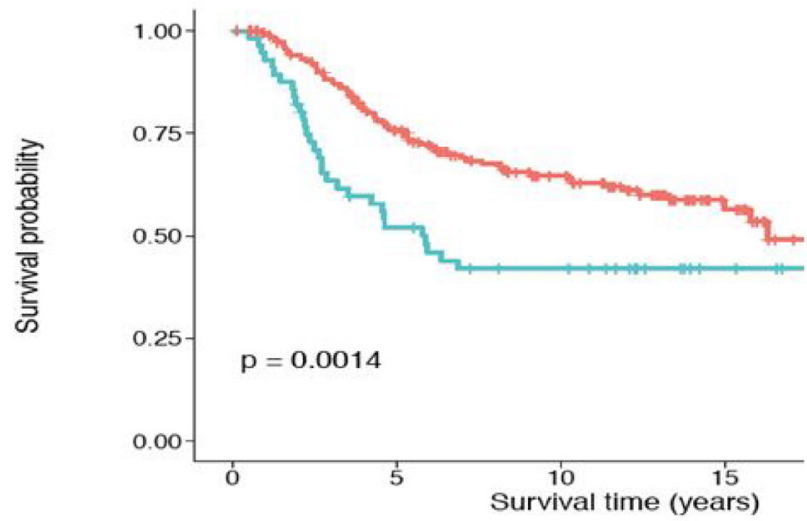

Number at risk by time

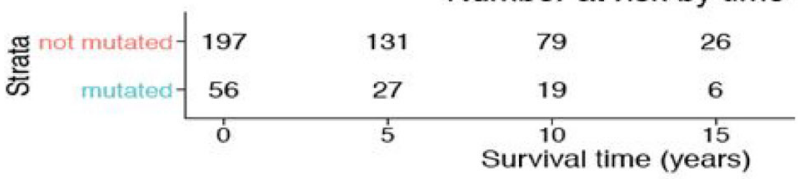

C

FFAR2

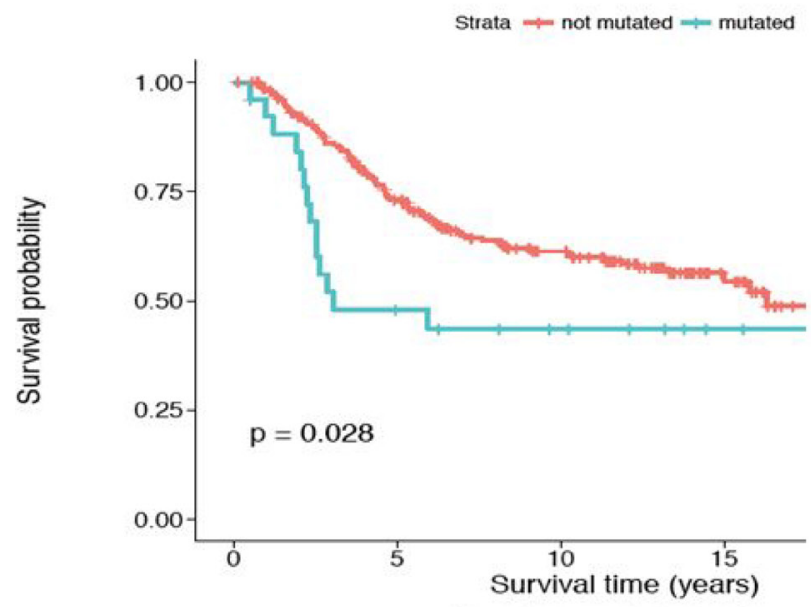

Number at risk by time

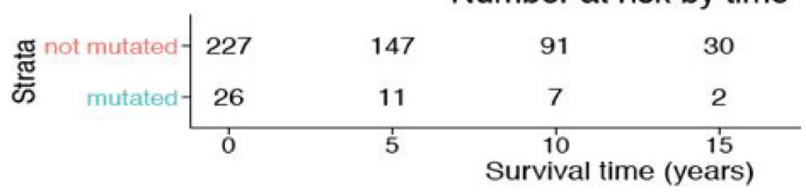

generated by PI3K and leads to activation of downstream signaling components. A well-known consequence is recruitment and activation of protein kinase Akt, which can phosphorylate a variety of substrates, which in turn activate cell growth, apoptosis and cell cycle processes. PIP3 is a substrate for phosphatase and tensin homologue (PTEN), which is required for dephosphorylation of PIP3 into PIP2,

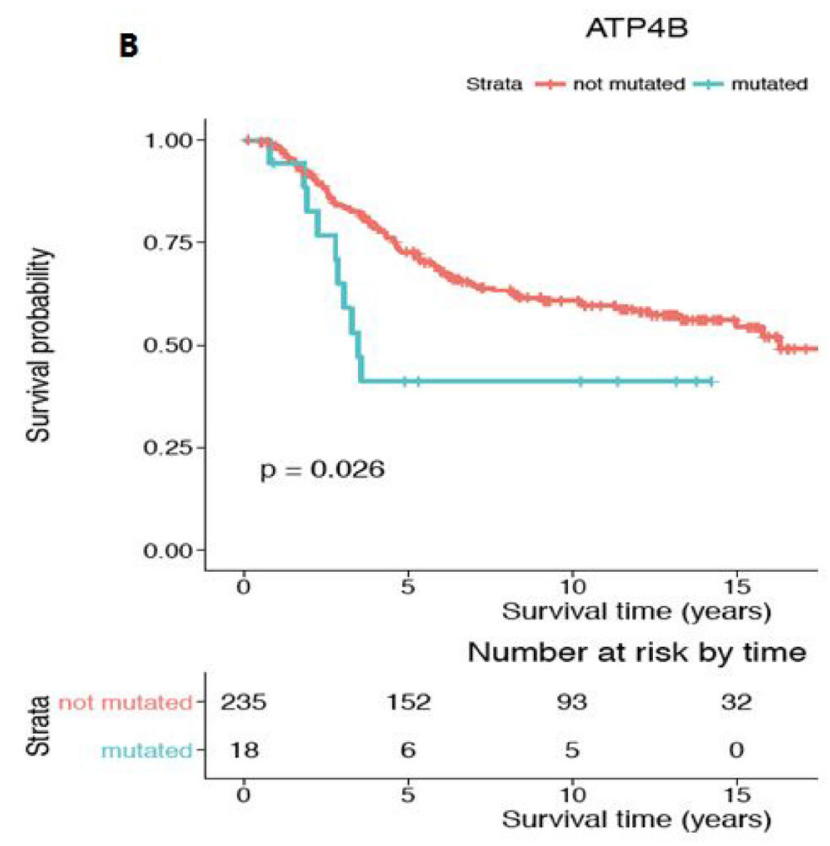

D

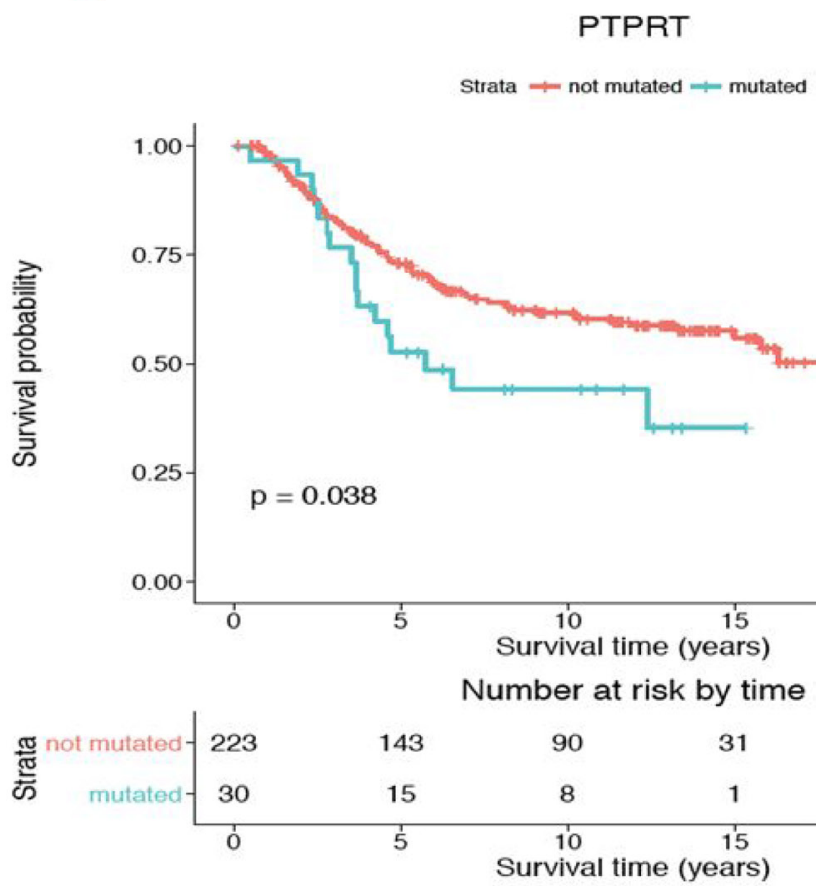

Figure 6: Kaplan-Meier survival analysis for genes with significant CNA gain mutations in the young women group. Genes showing with statistical significance $(p<0.05)$ are (A) ASB13, (B) ATP4B, (C) FFAR2 and (D) PTPRT. Survival curve in red represents patients without CNA mutation in the corresponding gene $(\mathrm{CN}=2)$ while the curve in blue represents patients with $\mathrm{CNA}$ gain mutations in the corresponding gene $(\mathrm{CN}>2)$. Y-axis is the cumulative survival probability and $\mathrm{X}$-axis is the survival time in years. 
A

PTENP1

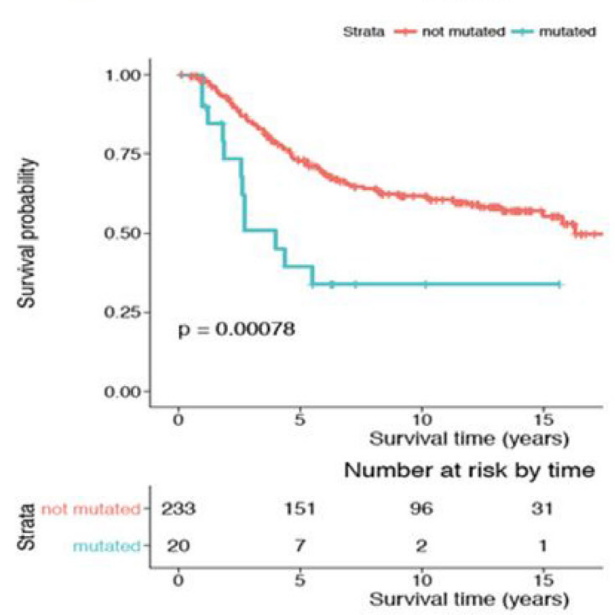

C

ZNF718

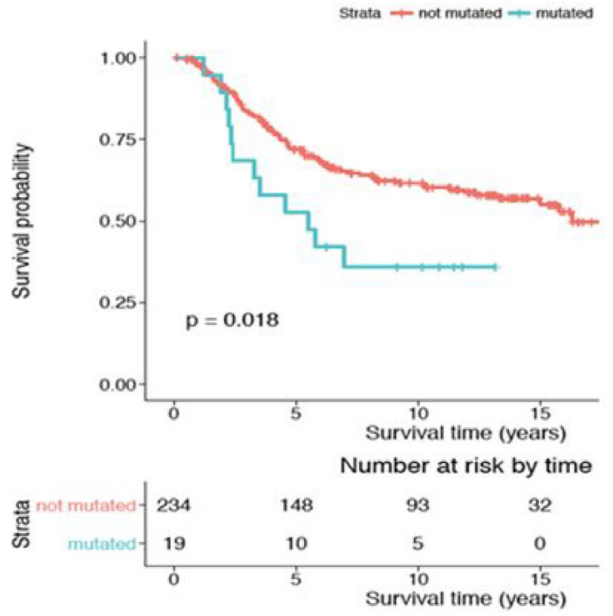

E
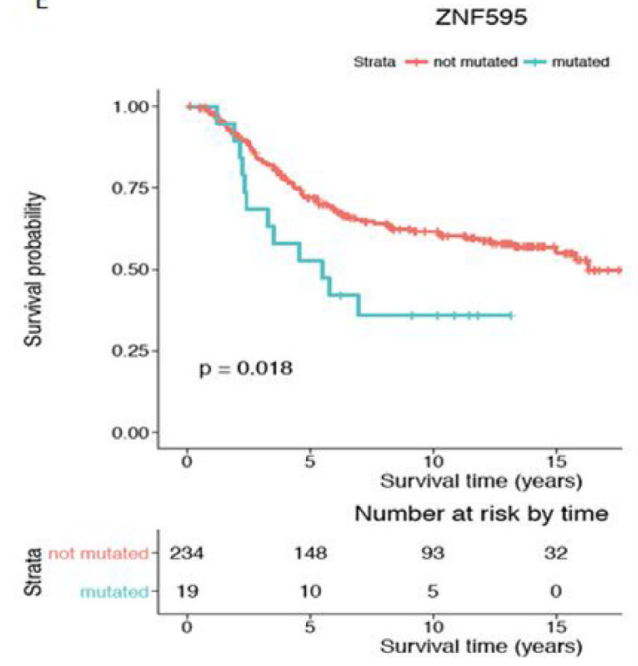
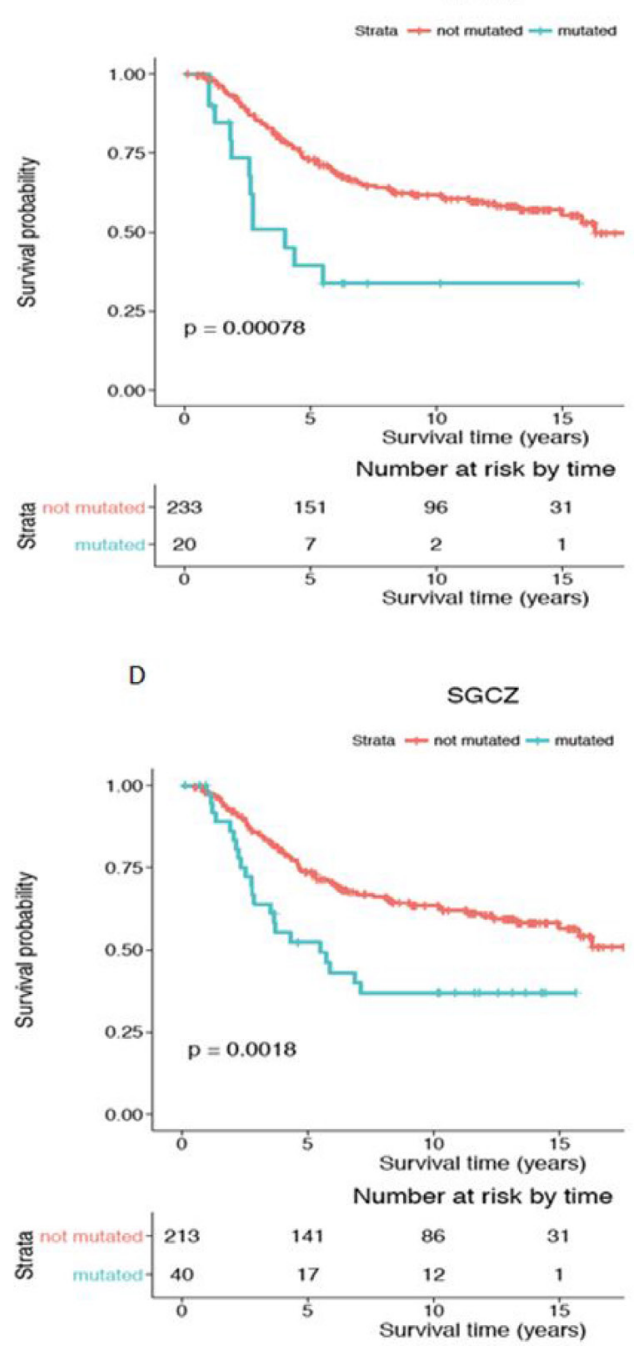

Figure 7: Kaplan-Meier survival analysis for genes with significant CNA loss mutations in the young women group. Genes showing statistical significance $(p<0.05)$ are (A) PTENP1, (B) PTEN, (C) ZNF718, (D) SGCZ and (E) ZNF595. Survival curve in red represents patients without CNA mutation in the corresponding gene $(\mathrm{CN}=2)$ while the curve in blue represents patients with CNA loss mutations in the corresponding gene $(\mathrm{CN}<2)$. Y-axis is the cumulative survival probability and $\mathrm{X}$-axis is the survival time in years. 
essential for inhibition of the AKT pathway. Dysregulation of these pathways is frequent in many cancer types.

\section{Cell adhesion}

Cellular adhesion plays a major role in maintaining the integrity of normal cell-cell connections, and disruption in this pathway has been strongly associated with metastasis in cancers. Adherens junctions, which are sites of intracellular signalling and anchoring, provide strong bonds between adjacent cell membranes. The molecular processes governing cell-cell adhesion are very finely controlled, since they inhibit epithelial-mesenchymal transition (EMT) that is normally present during embryogenesis and tissue repair. Characteristics of EMT include a loss in intercellular adhesion and enhancement of cell migration, leading to a more motile phenotype [32]. Notably, the adherens junctions are lost during the process of EMT, which increases the risk of cancer progression such as metastasis. In normal tissues, epithelial cells are tightly bound to one another. However, in advanced cancer, many epithelial tumour cells show loss of cell-cell adhesion and increased tissue invasion. Tumours featuring local spreading and invasion are suggested to have a more aggressive phenotype and be associated with a higher mortality rate of the patient. This phenomenon has been widely seen in various cancer types, including breast, colon, prostate, ovarian and other types of cancer [33].

\section{CONCLUSIONS}

Applying the graph-based algorithm to the Molecular Taxonomy of Breast Cancer International Consortium (METABRIC) breast cancer dataset, we have identified and validated 81 recurrent CNA gain regions and 25 validated recurrent CNA loss regions specific to young-Women's breast cancers. As well, we have located the corresponding candidate protein encoding genes that are encompassed in these regions. The graph-based algorithm guarantees that the identified CNA regions are the most frequent and that the minimal regions have been delineated.

Identification of molecular alterations associated with disease outcome may improve risk assessment and treatments for aggressive breast cancer, especially for young women. It can give new insights into the role of CNAs in cancer predisposition, development and progression as well as contribute to a more accurate and complete human cancer genome sequence reference. We hope that the results of this study will in the future, facilitate the development of screening methods for breast cancer biomarker discovery, especially in young women, as more prospective samples become available.

Since CNAs are fairly large in size, in the future it would be interesting to characterize further the noncoding CNA regions we have identified and their role in regulating gene expression levels either in cis or trans.

\section{MATERIALS AND METHODS}

\section{Data source}

All breast cancer data are retrieved from the Molecular Taxonomy of Breast Cancer International Consortium (METABRIC) [22], which is a novel dataset consisting of comprehensive clinical features such as breast cancer-specific survival data, PAM50 subtypes, ER/PR/HER2 status, tumour grade and tumour sizes. Each case has corresponding whole gene expression profiles (Illumina HT-12 v3 platform), SNPs and somatic DNA copy-number profiling data (Affymetrix Human SNP 6.0 platform). Treatments for the patients are homogeneous among each clinically relevant group: almost all ER-positive/LN-negative patients did not receive chemotherapy, while ER-negative/LN-positive patients did receive chemotherapy. Furthermore, the METABRIC cohort consists of cohorts prior to the usage of Herceptin/ trastazumab in standard clinical care. Therefore, the outcome of HER2 positive patients reflects the poor prognosis in such patients before the introduction of this targeted therapy [22].

All samples are derived from $\sim 2,000$ clinically annotated primary fresh-frozen breast cancer specimens from tumour banks in the UK and Canada (a discovery set of 997 primary tumours and a validation set of 995 tumours were divided by METABRIC). All genomic and clinically annotated data are available at the European Genome-Phenome Archive (http://www.ebi.ac.uk/ega/), under accession number EGAS00000000083 [22]. The individual CNA calls of the $\sim 2,000$ individual samples are pre-existing from the METABRIC study and downloaded from EGAS00000000083 [22]. Circular binary segmentation (CBS) method is used for making individual CNA calls. CBS is a segmentation-based method that scans for change points in an ordered sequence of copy number values to delineate segments with different distribution of the values (measured by having different means). In other words, it will recursively divide up the chromosome until segments that have probe distribution different than neighbours have been identified [41].

\section{Representing CNAs as an interval graph}

Figure 8A shows examples of five individual patient level CNA segments (CNA 1, CNA 2, CNA 3, CNA 4, CNA 5 ) on the same chromosome. Each of the five CNAs contains chromosomal-specific start (left) and end (right) positions. To identify the common regions of individual patient level CNAs on the same chromosome, the intersection among the individual patient level CNAs can be represented as an interval graph, treating each called individual patient level CNA as a vertex of the graph and connecting two vertices only if the corresponding intervals have an intersecting region. Thus, the constructed interval graph $G(V, E)$ is comprised of a set of 
vertices $V$, where each vertex $(v \in V)$ corresponds to a specific interval of the individual patient level CNA and each edge $(\{u, v\} \in E)$ connects two intersecting intervals $u$ and $v$. In Figure $8 \mathrm{~B}$, an example of the interval graph is shown where CNA 1 through CNA 4 are the intervals (nodes of the graph or individual patient level CNAs) and an edge connects two nodes (individual patient level CNAs) if the intervals overlap.

To find maximal cliques in an interval graph constructed from individual patient level CNAs, we applied Gentlemen and Vandal's algorithm [34]. The main idea of the algorithm is to sort the vertices based on their chromosomal end positions. The ordering is important because it allows the algorithm to discard vertices in each iteration without losing the triangulation property. The input of the algorithm is the individual patient level CNAs on a specific chromosome, which includes two parameters for each CNA segment: start and end positions (base pair).

Each of the identified maximal cliques is a recurrent CNA, which is common in multiple patients. The shared region of the recurrent CNA across multiple patients is the minimal common region (MCR) of the CNA, which has the potential to harbour cancer causing/associated genes. In practice, the size of the maximal cliques should be at least 2 and the size of the MCRs should be at least $1 \mathrm{~kb}$. It should be noted that we need to analyse CNA gains and losses separately. More details of the algorithm and its applications can be found in [35].

\section{Survival analysis}

Disease (breast cancer) specific survival analysis was performed for both the mutation status (CNA gain, CNA

CNA 4

CNA 3

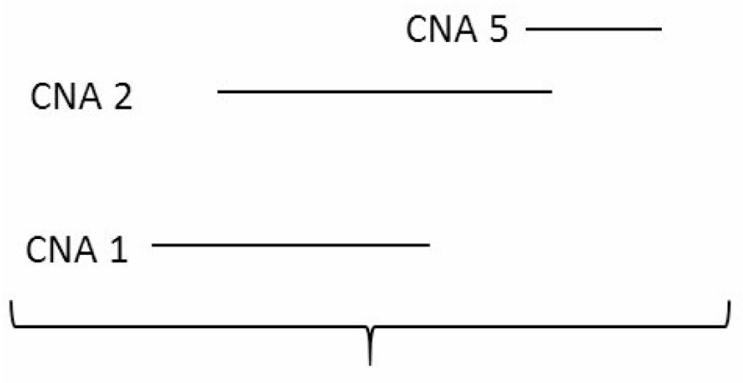

A: Called CNA loss) by the product-limit method or The Kaplan-Meier method and the expression level of the corresponding genes that are encompassed in the validated recurrent CNA regions using the Cox proportional hazard model [36]. All analyses were performed using Survival R package (https:// cran.r-project.org/web/packages/survival/index.html).

\section{eQTL analysis}

An expression quantitative trait locus (eQTL) is a locus that explains a portion of the genetic variance of a gene expression phenotype. An eQTL analysis tests for direct associations between markers of genetic variation with gene expression levels; that is, to evaluate the association between gene expression and CNA mutation status. Logistic regression is used to estimate the probability $\mathrm{p}$ associated with a dichotomous response for various values of an explanatory variable. In this case, the response (dependent) variable is gene expression (binarized-by-mean) and the predictor (independent) variable is CNA status.

\section{Functional analysis}

Functional analysis such as enrichment and annotations have been carried out using software (Enrichr and ANNOVAR) to determine whether the identified CNA regions with protein coding genes are enriched in any interesting pathways or functions. Enrichr software [37] contains a diverse and up-to-date collection of over 100 gene-set libraries available for analysis and download. It is used to perform pathway enrichment analysis on the
CNA $3 \quad$ CNA 2

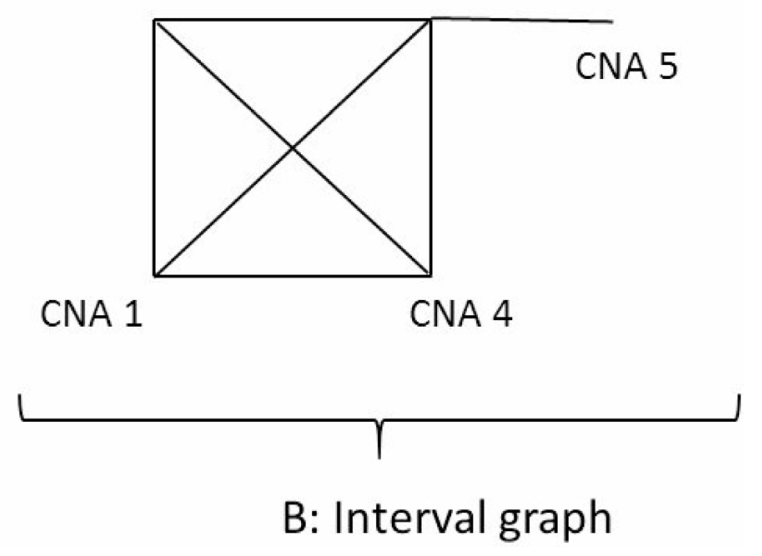

Figure 8: Representing CNAs as an interval graph. (A) CNA 1, CNA 2, CNA 3, CNA 4, CNA 5 are individual patient level CNAs on a specific chromosome. Each of the CNAs has chromosome start and end positions. (B) This is an interval graph where CNA 1, CNA 2, CNA 3, CNA 4, CNA 5 are the individual patient level CNAs in (A). The edge between each of two vertices in the graph represents the two individual patient level CNAs sharing a piece of common regions on the chromosome. 
identified young-specific genes to identify which pathways are over-represented in the gene-set. ANNOVAR [38] is a perl command line program for genome annotation. This region-based annotation is used to identify affected genomic regions that lie outside of the protein-coding regions.

\section{Biological visualization}

In order to aid in clearer visualization of and assist interpretation of the results, software programs Oncoprint [39] and CIMminer [40] were used to generate heatmap visualizations for the identified candidate regions. Oncoprint is included in the R package ComplexHeatMap, and it is a way to visualize multiple genomic alteration events in the format of a heatmap. This is used to visualize the frequencies of CNA mutation for each of the youngspecific regions with genes in Discovery and Validation datasets. CIMminer generates color-coded Clustered Image Maps (CIMs) to portray "high-dimensional" data sets such as gene expression profiles. It is used to visualize the relative expression levels in terms of colour intensity for each of the identified young-specific genes.

\section{Abbreviations}

CBS: Circular binary segmentation; ANNOVAR: Annotate Variation; BC: Breast cancer; CNA: Copy number alteration; CIM: Clustered Image Map; eQTL: Expression quantitative trait locus; EMT: Epithelialmesenchymal transition ; ER: Estrogen; GWAS: Genomewide association studies; METABRIC: Molecular Taxonomy of Breast Cancer International Consortium; PR: Progesterone receptor.

\section{Author contributions}

Conceived and designed the experiments: $\mathrm{PH}$. Analyzed the data: CC. Wrote the manuscript: CC. Contributed to the writing of the manuscript and interpreting the results: CC, PH, LCM. Agreed with manuscript results and conclusions: All authors. All authors reviewed and approved the final manuscript.

\section{ACKNOWLEDGMENTS}

The authors thank the Molecular Taxonomy of Breast Cancer International Consortium (METABRIC) for providing the data sets used in this study. The authors thank Dr. Kevin Coombs for providing insightful comments for the study.

\section{CONFLICTS OF INTEREST}

The authors declare no conflict of interest for this manuscript

\section{FUNDING}

This work was supported in part by Canadian Breast Cancer Foundation - Prairies/NWT Chapter, Natural Sciences and Engineering Research Council of Canada, Manitoba Research Health Council and University of Manitoba. The authors confirm that the funder had no influence over the study design, content of the article, or selection of this journal.

\section{REFERENCES}

1. Anders CK, Johnson R, Litton J, Phillips M, Bleyer A. Breast cancer before age 40 years. Semin Oncol. 2009; 36:237-249.

2. Perou CM, Sørlie T, Eisen MB, van de Rijn M, Jeffrey SS, Rees CA, Pollack JR, Ross DT, Johnsen H, Akslen LA, Fluge O, Pergamenschikov A, Williams C, et al. Molecular portraits of human breast tumours. Nature. 2000; 406:747-752.

3. Bauer KR, Brown M, Cress RD, Parise CA, Caqqiano V. Descriptive analysis of estrogen receptor (ER)-negative, progesterone receptor (PR)-negative, and HER2-negative invasive breast cancer, the so-called triple-negative phenotype: a population-based study from the California cancer Registry. Cancer. 2007; 109:1721-1728.

4. Azim HA Jr, Michiels S, Bedard PL, Singhal SK, Criscitiello C, Ignatiadis M, Haibe-Kains B, Piccart MJ, Sotiriou C, Loi S. Elucidating prognosis and biology of breast cancer arising in young women using gene expression profiling. Clin Cancer Res. 2012; 18:1341-1351.

5. Gnerlich JL, Deshpande AD, Jeffe DB, Sweet A, White N, Margenthaler JA. Elevated breast cancer mortality in women younger than age 40 years compared with older women is attributed to poorer survival in early-stage disease. J Am Coll Surg. 2009; 208:341-347.

6. Johnson RH, Hu P, Fan C, Anders CK. Gene expression in "young adult type" breast cancer: a retrospective analysis. Oncotarget. 2015; 6:13688-13702. https://doi.org/10.18632/ oncotarget.4051.

7. Bharat A, Aft RL, Gao F, Margenthaler JA. Patient and tumor characteristics associated with increased mortality in young women ( $\leq 40$ years) with breast cancer. J Surg Oncol. $2009 ; 100: 248-251$.

8. Futreal PA, Coin L, Marshall M, Down T, Hubbard T, Wooster R, Rahman N, Stratton MR. A census of human cancer genes. Nat Rev Cancer. 2004; 4:177-183.

9. Beroukhim R, Mermel CH, Porter D, Wei G, Raychaudhuri S, Donovan J, Barretina J, Boehm JS, Dobson J, Urashima M, McHenry KT, Pinchback RM, Ligon AH, et al. The landscape of somatic copy-number alteration across human cancers. Nature. 2012; 463:899-905.

10. Stuart D, Sellers WR. Linking somatic genetic alterations in cancer to therapeutics. Curr Opin Cell Biol. 2009; 21:304-310. 
11. Baudis M. Genomic imbalances in 5918 malignant epithelial tumors: an explorative meta-analysis of chromosomal CGH data. BMC Cancer. 2007; 7:226.

12. Weir BA, Woo MS, Getz G, Perner S, Ding L, Beroukhim R, Lin WM, Province MA, Kraja A, Johnson LA, Shah K, Sato M, Thomas RK, et al. Characterizing the cancer genome in lung adenocarcinoma. Nature. 2007; 450:893-898.

13. Eder AM, Sui X, Rosen DG, Nolden LK, Cheng KW, Lahad JP, Kango-Singh M, Lu KH, Warneke CL, Atkinson EN, Bedrosian I, Keyomarsi K, Kuo WL, et al. Atypical PKCiota contributes to poor prognosis through loss of apical-basal polarity and cyclin E overexpression in ovarian cancer. Proc Natl Acad Sci USA. 2005; 102:12519-12524.

14. Zender L, Spector MS, Xue W, Flemming P, CordonCardo C, Silke J, Fan ST, Luk JM, Wigler M, Hannon GJ, $\mathrm{Mu} \mathrm{D}$, Lucito R, Powers $\mathrm{S}$, et al. Identification and validation of oncogenes in liver cancer using an integrative oncogenomic approach. Cell. 2006; 125:1253-1267.

15. Chitale D, Gong Y, Taylor BS, Broderick S, Brennan C, Somwar R, Golas B, Wang L, Motoi N, Szoke J, Reinersman JM, Major J, Sander C, et al. An integrated genomic analysis of lung cancer reveals loss of DUSP4 in EGFR mutant tumors. Oncogene. 2009; 28:2773-2783.

16. Cancer Genome Atlas Research Network. Comprehensive genomic characterization defines human glioblastoma genes and core pathways. Nature. 2008; 455:1061-1068.

17. Chapman PB, Hauschild A, Robert C, Haanen JB, Ascierto P, Larkin J, Dummer R, Garbe C, Testori A, Maio M, Hogg D, Lorigan P, Lebbe C, et al. Improved survival with vemurafenib in melanoma with BRAF V600E mutation. New England Journal of Medicine. 2011; 364:2507-2516.

18. Nguyen KS, Neal JW. First-line treatment of EGFR-mutant non-small-cell lung cancer: the role of erlotinib and other tyrosine kinase inhibitors. Biologics. 2012; 6:337-345.

19. Phillips JL, Hayward SW, Wang Y, Vasselli J, Pavlovich C, Padilla-Nash H, Pezullo JR, Ghadimi BM, Grossfeld GD, Rivera A, Linehan WM, Cunha GR, Ried T. The consequences of chromosomal aneuploidy on gene expression profiles in a cell line model for prostate carcinogenesis. Cancer Res. 2001; 61:8143-8149.

20. Pollack JR, Sørlie T, Perou CM, Rees CA, Jeffrey SS, Lonning PE, Tibshirani R, Botstein D, Børresen-Dale AL, Brown PO. Microarray analysis reveals a major direct role of DNA copy number alteration in the transcriptional program of human breast tumors. Proc Natl Acad Sci USA. 2002; 99:12963-12968.

21. Parker JS, Mullins M, Cheang MC, Leung S, Voduc D, Vickery T, Davies S, Fauron C, He X, Hu Z, Quackenbush JF, Stijleman IJ, Palazzo J, et al. Supervised risk predictor of breast cancer based on intrinsic subtypes. J Clin Oncol. 2009; 27:1160-1167.
22. Curtis C, Shah SP, Chin SF, Turashvili G, Rueda OM, Dunning MJ, Speed D, Lynch AG, Samarajiwa S, Yuan Y, Gräf S, Ha G, Haffari G, et al. The genomic and transcriptomic architecture of 2,000 breast tumours reveals novel subgroups. Nature. 2012; 486:346-352.

23. Bose S, Wang SI, Terry MB, Hibshoosh H, Parsons R. Allelic loss of chromosome 10q23 is associated with tumor progression in breast carcinomas. Oncogene. 1998; 17:123-127.

24. Feilotter HE, Coulon V, McVeigh JL, Boag AH, DorionBonnet F, Duboue B, Latham WC, Eng C, Mulligan LM, Longy M. Analysis of the 10q23 chromosomal region and the PTEN gene in human sporadic breast carcinoma. Br J Cancer. 1999; 79:718-723.

25. Zhou X, Thorgeirsson SS, Popescu NC. Restoration of DLC-1 gene expression induces apoptosis and inhibits both cell growth and tumorigenicity in human hepatocellular carcinoma cells. Oncogene. 2004; 23:1308-13.

26. Emi M, Utada $Y$, Yoshimoto $M$, Sato $T$, Minobe $K$, Matsumoto S, Akiyama F, Iwase T, Haga S, Kajiwara T, Sakamoto G. Correlation of allelic loss with poor postoperative survival in breast cancer. Breast Cancer. 1999; 6:351-6.

27. Ho WC, Pikor L, Gao Y, Elliott BE, Greer PA. Calpain 2 regulates Akt-FoxO-p27Kip1 protein signaling pathway in mammary carcinoma. Journal of Biological Chemistry. 2012; 287:15458-65.

28. Whitaker HC, Shiong LL, Kay JD, Grönberg H, Warren AY, Seipel A, Wiklund F, Thomas B, Wiklund P, Miller JL, Menon S. N-acetyl-L-aspartyl-L-glutamate peptidaselike 2 is overexpressed in cancer and promotes a promigratory and pro-metastatic phenotype. Oncogene. 2014; 33:5274-87.

29. Menendez JA, Lupu R. Fatty acid synthase and the lipogenic phenotype in cancer pathogenesis. Nature Reviews Cancer. 2007; 7:763-77.

30. Wymann MP, Schneiter R. Lipid signalling in disease. Nature Reviews Molecular Cell Biology. 2008; 9:162-176.

31. Lingwood D, Simons K. Lipid rafts as a membraneorganizing principle. Science. 2010; 327:46-50.

32. Talbot LJ, Bhattacharya SD, Kuo PC. Epithelialmesenchymal transition, the tumor microenvironment, and metastatic behavior of epithelial malignancies. Int $\mathrm{J}$ Biochem Mol Biol. 2012; 3:117-136.

33. Martin TA, Mansel RE, Jiang WG. Loss of occludin leads to the progression of human breast cancer. Int J Mol Med. 2010; 26:723-734.

34. Gentleman R, Vandal C. Computational Algorithms for Censored-Data Problems Using Intersection Graphs. J Comput Graph Stat. 2001; 10:403-421.

35. Chi C, Ajwad R, Kuang Q, Hu P. A graph-based algorithm for detecting recurrent copy number variants in cancer studies. Cancer Informatics. 2016; 2:43-50.

36. Therneau T, Grambsch P. Modeling Survival Data: Extending the Cox Model. New York: Springer-Verlag. 2000. 
37. Chen EY, Tan CM, Kou Y, Duan Q, Wang Z, Meirelles GV, Clark NR, Ma'ayan A. Enrichr: interactive and collaborative HTML5gene list enrichment analysis tool. BMC Bioinformatics. 2013; 14:128.

38. Wang K, Li M, Hakonarson H. ANNOVAR: functional annotation of genetic variants from high-throughput sequencing data. Nucleic Acids Research. 2010; 38:e164.

39. Cerami E, Gao J, Dogrusoz U, Gross BE, Sumer SO, Aksoy BA, Jacobsen A, Byrne CJ, Heuer ML, Larsson E, Antipin Y, Reva B, Goldberg AP, et al. The cBio cancer genomics portal: an open platform for exploring multidimensional cancer genomics data. Cancer Discovery. 2012; 2:401-404.

40. Weinstein JN, Myers TG, O'Connor PM, Friend SH, Fornace AJ Jr, Kohn KW, Fojo T, Bates SE, Rubinstein LV, Anderson NL, Buolamwini JK, van Osdol WW, Monks AP, et al. An information-intensive approach to the molecular pharmacology of cancer. Science. 1997; 275:343-349.

41. Erdman C, Emerson JW. A fast Bayesian change point analysis for the segmentation of microarray data. Bioinformatics. 2008; 24:2143-2148. 